\title{
VIOLENCIA DOMÉSTICA EN FINLANDIA Y EN ESPAÑA
}

\author{
CARMEN PÉREZ BELDA
}

Estudiante Doctorado. Universidad de Laponia, Rovaniemi (Finlandia).

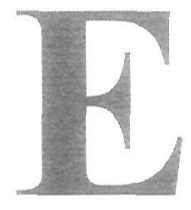

\section{ABSTRACT}

n la actualidad y debido a la europeización, internacionalización y globalización, la gente está más interesada en comparaciones internacionales y, lo que es más importante, en tener un mejor acceso y facilidades para comparar la realidad en diferentes países. El siguiente artículo está basado en un estudio comparativo sobre la situación de la violencia doméstica en Finlandia y en España realizado en la Universidad de Laponia en Rovaniemi, con su apoyo y el del Centro Móvil (Centro de crisis, Albergue y Hogar para madres e hijos) de Rovaniemi, donde realicé mis prácticas y tuve la oportunidad de trabajar directamente con un caso de violencia doméstica.

Tras una breve conceptualización del problema, se destacan tres aspectos importantes para destacar las diferencias existentes entre ambos países. En primer lugar, una pequeña revisión histórica acerca de las principales diferencias de los modelos de estados de bienestar en Finlandia y en España. En segundo lugar, se analiza y compara la influencia del estado de bienestar en el desarrollo de políticas sociales en contra de la violencia doméstica basado en la comparación estadística sobre el problema en ambos países. En tercer lugar, se analiza y compara el tratamiento de la violencia doméstica en Finlandia y en España.

\section{INTRODUCCIÓN. ¿QUÉ ENTENDEMOS POR VIOLENCIA DOMÉSTICA?}

La violencia doméstica supone un grave problema a nivel mundial. Las mujeres y niñas siguen sufriendo cada día violencia a manos de sus maridos, compañeros, padres y hermanos, con independencia de su nivel cultural, económico y social. La violencia contra las mujeres es una manifestación de relación de poder, históricamente desigual, del hombre sobre la mujer, impidiendo de este modo su desarrollo personal. 
Durante las últimas décadas, la violencia doméstica ha sido analizada desde muchas perspectivas, judicial, legal, incluyendo la discusión a cerca de la naturaleza de género y relaciones de género, y muchas publicaciones y artículos han aparecido sobre este tema. Éste no es un fenómeno nuevo, estudios recientes revelan que es un problema que está alcanzando proporciones epidémicas. Existe una aceptación histórica de los derechos del marido o compañero para dominar y abusar de su mujer o compañera, la legislación y muchas de las instituciones, incluyendo los medios de comunicación, subscriben dicha aceptación. En muchas sociedades las mujeres han sido consideradas tradicionalmente como propiedad del hombre, marido o padre; el deber del hombre era enseñarles disciplina a la mujer y a los hijos e hijas a través de golpes (Summers \& Hoffman, 2002).

La intimidad en las relaciones de pareja enmascara a través de la palabra "doméstica" una gran variación en la calidad de la relación, pues violencia doméstica no es confinada solamente dentro del hogar, puede ser perpetrada en muchas localizaciones geográficas:

El abuso contra mujeres es raramente discutido. Ocurre en secreto y debido a que la mujer normalmente vive con el hombre maltratador, raramente alguien fuera del ámbito del hogar sabe de la situación de violencia que está sufriendo la victima. La mayoría de la gente no parece darse cuenta de la cantidad de personas que están siendo deprivadas de sus derechos básicos (Horley, 1988,1).

¿Qué entendemos por violencia doméstica? Entendemos por violencia doméstica cualquier tipo de violencia referida a cualquier clase de maltrato físico, emocional o sexual, perpetrado dentro de la esfera familiar hacia la mujer, niños o personas mayores. Por otro lado, la expresión de violencia de género se refiere a acciones de abuso contra las mujeres, simplemente por el hecho de serlo. Dichas acciones son realizadas por el hombre con quien la víctima mantiene una relación afectiva de pareja, una relación laboral o con alguien con quien no tiene ningún tipo de relación (Manual sobre violencia contra la mujer 2001, 20-21). Otros autores autores hacen referencia a la relación abuso por parte de los maltratadores, como Pryke \& Thomas $(1998,40)$ :

La relación de abuso envuelve en esencia el abuso de poder. Esto incluye violencia, abuso e intimidación, violencia sexual, emocional, mental o económica contra la persona o sus propiedades, infringido por un adulto conocido $u$ otra persona (normalmente su pasada o actual pareja), perteneciente al hogar o externa al mismo. El maltratador tiene la opción (reconocida o no) de ejercer o no su poder y control 
sobre la otra persona pero cada persona tiene el derecho de estar libre del miedo y/o del abuso y de la naturaleza potencial y criminal de semejantes ofensas que deben ser reconocidas.

Algunos autores han preferido ver la violencia doméstica como una manifestación mucho más amplia de violencia familiar. Según Dobash \& Dobash $(1992,239)$ :

... Es asumido que el interés, acciones y creencias de todos los miembros de la familia son idénticos, ignorando el cúmulo de experiencias que demuestran la diferencia que al mismo tiempo existen distintos mundos e intereses de hombres, mujeres y niños... la perspectiva de los sistemas familiares ha sido criticada por su acercamiento neutral de género, aislamiento en la investigación sobre la mujer, un fracaso a la hora de considerar poder y un más amplio contexto sociocultural, el uso de un abstracto, "neutral" lenguaje y contradicción, tienden a culpar a las madres e idealizar a los padres.

Sin embargo, un concepto que ha sido visto como una explicación parcial de por qué algunas mujeres continúan en una relación de malos tratos durante mucho tiempo es el denominado "el síndrome de la mujer maltratada". Es la decisión de la mujer de volver con su pareja maltratadora, particularmente cuando ella ha vuelto anteriormente en otras ocasiones. Este tipo de comportamiento debe ser explicado a través de otros factores, por ejemplo:

- Es posible que tenga que hacer frente a grandes dificultades a la hora de encontrar un hogar alternativo y seguro.

- Es posible que tenga problemas económicos, y sienta una ambigüedad considerable sobre el hombre con quien ha vivido durante años (y que ahora esta totalmente arrepentido y prometiendo que su comportamiento cambiará).

- Es posible que tenga presiones desde la familia de origen para intentar arreglar la situación por los niños, pues los niños desearán ver a su padre.

- Es posible incluso que ella haya continuado sintiendo inseguridad al querer escapar de la situación y sienta menos riesgo con la opción de volver con él (Pryke \&Thomas 1998, 44). 


\section{REVISIÓN HISTÓRICA DEL ESTADO DE BIENESTAR EN FINLANDIA Y ESPAÑA.}

¿Cómo se percibe algo explicado en palabras para comprender cómo la tradición histórica, cultural y política ha rebotado en el desarrollo de la violencia doméstica en dos diferentes países como Finlandia y España? Estudios y comparaciones entre países pueden revelar importantes "insights" en cuestiones de similitudes y diferencias y modelos de convergencia o diversidad.

\subsection{El caso de Finlandia.}

En 1906, la mujer finlandesa fue la primera mujer en toda Europa en recibir el derecho a voto y la primera mujer en el mundo que fue elegible para las elecciones del parlamento. En aquel tiempo Finlandia era uno de los países más agrícolas de toda Europa. La paradoja fue que el insofisticado nivel de la sociedad fue lo que facilitó la aceptación de la idea que los derechos políticos debían de ser extendidos a las mujeres. La mujer trabajó igual o más que los hombres en las granjas además de tareas familiares. A comienzos del siglo veinte, tres cuartos de todas las mujeres trabajaban en el campo. La agricultura no perdió su protagonismo como principal recurso de empleo en Finlandia hasta los años cincuenta.

Desde la Segunda Guerra Mundial, la producción femenina, particularmente la mujer casada, en la fuerza de trabajo se incrementó. El incremento de la participación de la mujer en la vida laboral no significó, sin embargo, que hubiera igualdad entre los sexos en el mercado laboral. Existe todavía una clara división entre trabajos de hombres o de mujeres, aunque esta frontera ha comenzado a desaparecer en los recientes años. Los salarios de las mujeres en ambos sectores, público y privado, son todavía solamente el $80 \%$ del salario de los hombres aunque las mujeres de un mismo grupo de edad tienen mayor educación que los hombres.

Sin embargo, y a pesar de las posibles desigualdades entre hombres y mujeres existentes todavía, Finlandia, al igual que el resto de países nórdicos, es asociado con un Estado de bienestar institucional, universal y social-demócrata. Finlandia tuvo un tardío comienzo en su desarrollo industrial comparativamente con el resto de países nórdicos, pues es un país independiente sólo desde 1917. Su modelo de bienestar ha sido caracterizado por el desarrollo de la seguridad social al igual como la autonomía de cada individuo, debido al elevado nivel de intervención pública en la economía y la distribución del apoyo a individuos como tales más que a la familia como institución. 
Según Esping-Andersen (1990), las claves del Estado de Bienestar están en relación con el Estado y el mercado, la estratificación y la desmercantilización, donde el estado es al mismo tiempo un sistema de estratificación y un mecanismo de intervención en la estructura de la desigualdad. En este caso, Finlandia formaría parte del grupo de los países social-demócratas, basado en el universalismo y desmercantilización de los derechos sociales, siendo el primer impulso de la reforma social, buscando la igualdad en el más elevado nivel y no un mínimo de igualdad en la mínimas necesidades, donde las clases medias son muy importantes en la consolidación del Estado de Bienestar después de la Segunda Guerra Mundial debido a la política de alianzas.

\subsection{El caso de España.}

Sin embargo, en España la historia es diferente y por lo tanto también el desarrollo del modelo de Estado de Bienestar. En España el comienzo de la industrialización llegó durante el periodo de la Restauración y sólo en determinadas zonas, como Cataluña, Vizcaya y Madrid, ésta última como el núcleo de servicios y capital financiera. Las diferencias de clases en la sociedad española han estado marcadas históricamente y la aceptación de universalidad de derechos sociales no llegó a la población. Todo ello, sumado a los cuarenta años de dictadura franquista retrasó la creación de un Estado de Bienestar, por lo tanto, en el Estado de Bienestar español, que según Jones (1993) formaría parte de la denominada "Franja Latina", el derecho al bienestar es caracterizado por su residualismo y la "entrada" obligada en el mercado de trabajo; ciertos programas de Seguridad Social cumplen la función de suministrar una renta básica aunque no estén diseñados como tales; no tener tradición de pleno empleo y haber realizado fuertes promesas de bienestar aunque falla el desarrollo social, institucional y legal (Picó, 1996).

Más explícitamente, se puede decir que España, un Estado de composición plural, asistió en la última parte del siglo XX a la federalización de su organización política territorial (Moreno, 1997ª). Y desde el inicio de la institucionalización, en 1978 con la Constitución y hasta 1995, la descentralización competencial del Estado de las Autonomías se ha traducido en una modificación sustancial de la participación de los tres niveles gubernamentales en el gasto público.

En los escenarios futuros del desarrollo de bienestar en España, el papel a desempeñar por las comunidades autónomas y los municipios será, por tanto, fundamental. Sin embargo, en España las responsabilidades en la actuación de los municipios aún resta por encajarse, mientras el protagonismo de los niveles intermedios de gobierno aumenta. A 
menudo sucede que la transferencia de responsabilidades y competencias a las administraciones locales no va acompañada de las adecuadas dotaciones presupuestarias. Por lo tanto trabajadores sociales de base se encuentran inmersos en actuaciones mediatizadas por unos objetivos contradictorios. Además la actuación complementaria de las asociaciones del tercer sector depende en muchos casos de los recursos públicos transferidos localmente, lo que puede suponer que la sociedad civil corra el riesgo de pasar de ser subsidiaria a estar subsidiada, y los trabajadores sociales deban desplegar una capacidad doble de coordinación y supervisión (Moreno, 2001, 79).

\section{III. ¿CÓMO INFLUYEN LOS DIFERENTES ESTADOS DE BIEN- ESTAR Y LAS POLÍTICAS SOCIALES EN LA SITUACIÓN DE VIOLENCIA DOMÉSTICA ENTRE AMBOS PAÍSES?}

En Finlandia, la ausencia de un tradicional rol masculino previno el desarrollo de una cultura e instituciones fuertemente patriarcales. En 1974, se inicia la separación de impuestos y, desde entonces, los beneficios de la seguridad social van destinados directamente hacia las mujeres como personas (como ciudadanas, trabajadoras, madres), pero no como esposas. La estabilización del trabajo remunerado para las mujeres es importante para la seguridad del Estado de Bienestar, sin embargo, Finlandia es el país nórdico más conservador en relación con el resto. Por ejemplo, la violación dentro del matrimonio fue criminalizada solamente en 1994 y la violencia familiar fue solamente un asunto de la fiscalía en 1995. Existen también otros indicadores de actitud patriarcal en Finlandia. El derecho de la mujer de conservar su apellido no llegó hasta 1980. Las relaciones de parejas homosexuales han sido legalizadas en 2002 (http:://www.virtual.finland.fi/English/women/women.html).

Por otro lado, en la sociedad finlandesa, los jóvenes se independizan a una edad mucho más temprana que en España, sobre los 20 años. El presente elevado nivel de ayudas de la seguridad social ayuda a los jóvenes a independizarse. La sociedad proporciona apoyo económico para todos lo jóvenes a partir de los 20 años de edad que abandonen el hogar familiar, independientemente del nivel económico de los padres. Para los estudiantes universitarios esto ocurre incluso antes, por ejemplo, tan pronto como comienzan a estudiar, dependiendo de su situación económica.

La cohabitación en pareja es muy popular entre los jóvenes finlandeses. La cohabitación en los apartamentos de estudiantes es el primer paso para los jóvenes a la hora de comenzar oficialmente una relación estable. Resulta extraño que una pareja joven se case sin ha- 
ber convivido antes. Por lo tanto, el número de niños nacidos fuera del matrimonio también está aumentando en más de un tercio. En el año 2000 , menos de la mitad de las mujeres que tuvieron su primer hijo estaban casadas y en una décima parte del total de niños nacidos, sus madres no tenían una relación permanente y estable. Este número ha aumentado en los últimos años, en la mayoría de las parejas con niños su paternidad es reconocida ante las autoridades. Al mismo tiempo, cuando se casan, esta convirtiéndose en "moda" realizar grandes celebraciones. Pero la razón por la cual las mujeres finlandesas tienen sus hijos y se casan tarde es debido al alto nivel de educación. Actualmente, las mujeres jóvenes tienen mayor educación que los hombres (http:/ /www.virtual.finland/English/women/women.html).

Esta temprana introducción a la vida en pareja nos ayuda a explicar la diferencia existente entre España y Finlandia en la edad de las víctimas. En Finlandia el grupo de mujeres con edades comprendidas entre los 25 a los 44 es el más alto dentro de la estadística de las edades de las víctimas que sufren maltratos. Sin embargo, en España encabeza la lista las mujeres con edades comprendidas entre los 40 hasta los 64 años. Esto se relaciona con la edad de emancipación en España que se sitúa alrededor de los 30 años. En ambos casos es el marido o compañero el principal maltratador.

Muchas razones relacionadas entre sí influyen en este hecho. En primer lugar el desempleo de larga duración y precariedad, y falta de calidad laboral suponen el primer obstáculo a la hora de independizarse. A ello se suma la falta de una política de vivienda que facilite el acceso de los jóvenes a la adquisición de la misma, además de la promoción y acceso a la vivienda en alquiler. El alto coste de las viviendas y la baja inversión del Estado en la promoción de viviendas sociales influye directamente en la permanencia de los jóvenes españoles en el hogar familiar de los padres. Esto a su vez acarrea otro tipo de consecuencias como el retraso de la entrada al matrimonio y en la edad de tener el primer hijo y descenso en la natalidad, hasta tal punto que España ha llegado a convertirse en el país con la tasa más baja de natalidad de Europa. Aunque en los últimos años se ha percibido un pequeño ascenso en las estadísticas debido a la inmigración.

También es importante destacar en cuanto a los grupos de mujeres divididas por edades y según las estadísticas, que un $17 \%$ de las mujeres españolas sufren malos tratos entre 26 y 35 años y un $16 \%$ durante menos de 5 años. La diferencia tan grande existente entre el primer y segundo caso podría deberse a que en el primero se trate posiblemente del primer matrimonio de la mujer y ésta se haya dedicado siempre al hogar mientras que el segundo grupo podría tratarse de mujeres que 
están comenzando una nueva relación. En España solamente el $31 \%$ de las víctimas trabajan, y más del $40 \%$ con un nivel de estudios primarios.

Todos los datos citados anteriormente nos hacen tener un perfil de la mujer víctima de malos tratos en España como mujer entre 44 y 64 años, que lleva más de 26 años viviendo con su pareja, con un nivel de estudios primarios y sin trabajo, dependiente totalmente de su marido y agresor.

Por otro lado la tasa de nacimientos es alto en Finlandia, comparado con el resto de países del Oeste de Europa. La legislación laboral provee a las madres con una garantía contra el despido de trabajo durante el permiso por maternidad. El sustento de la familia es asegurado por largos periodos de permiso de maternidad pagada, nueve meses, de los que los tres primeros van obligatoriamente dirigidos a la madre y el resto pueden ser disfrutados por la madre o por el padre. Además, uno de los padres tiene el derecho a salir del trabajo para ver al niño/a hasta la edad de tres años, aunque estas salidas no son pagadas.

La proporción de todas las familias con niños representados por segundas familias ha permanecido más o menos estable y se sitúa en un siete por ciento. Después que una familia se rompe, el umbral para establecer una nueva familia es relativamente alto. Finlandia y Suecia están a la cabeza en la estadística de divorcios entre todos los países de la Unión Europea. La Ley del Matrimonio de 1988 facilita la disolución del matrimonio. No es necesario identificar una parte culpable para obtener el divorcio, o un acuerdo de varias sesiones obligatorias para la tentativa de reconciliación. Hoy en día, solamente se requiere la solicitud de uno de los esposos para divorciarse, y después de un periodo de seis meses (periodo de reconsideración) el divorcio es oficial y, si una de las partes no está de acuerdo, no puede hacer nada para impedirlo. Las estadísticas indican que casi la mitad de los matrimonios acabarán en divorcio, a menos que la presente tendencia cambie.

Por lo tanto, el sentimiento de independencia en la sociedad finlandesa está muy marcado. Una mujer finlandesa gana su propio sustento, independientemente que el nivel de seguridad social sea suficientemente alto como para que una madre soltera no necesite el soporte del hombre para vivir. La sociedad garantiza una paga de alimentación, de modo que si el padre no lo hace, la seguridad social pagará una suma mínima acordada e intenta recuperar los atrasos del padre.

Todos los padres con hijos o hijas menores de 17 años reciben una prestación mensual por cada hijo/a indiferentemente de la situación económica de éstos. Esta prestación es un tercio más elevada si es un 
padre o madre soltero/a. En el pasado, las madres con frecuencia solían estar en casa cuando sus niños nacían, pero desde 1970s la situación ha cambiado y las madres van a trabajar. Un eficiente sistema de cuidados de día permite a los padres trabajar.

Una pre-escuela es ofertada a un precio muy razonable. Guarderías públicas, centros de día o familias cuidadoras son servicios supervisados por las autoridades locales. Incluso los niños con padres desempleados tiene ese derecho. Para padres con insuficientes medios, este tipo de servicios es gratuito. Los padres con hijos/as en edad pre-escolar tienen derecho a una semana de trabajo más corta, aunque no todo el mundo utiliza este privilegio.

Los niños en Finlandia no comienzan el colegio hasta los siete años de edad. En la escuela la comida es gratis, cosa que ayuda, además de económicamente, a conciliar la vida laboral de los padres con el cuidado de los hijos. Este servicio es ofrecido hasta la finalización de la educación secundaria o educación vocacional (formación profesional). (http:/ /www.virtual.finland.fi/English/women/women.html).

Este tipo de prestaciones públicas en Finlandia suponen un gran apoyo y seguridad por parte de las mujeres en una situación de violencia doméstica a la hora de tomar la decisión de abandonar el hogar y el agresor, porque saben que el Estado les proporcionará el apoyo suficiente para poder vivir independiente y con sus hijos. Todo ello, además de los servicios de urgencia y asistencia que en situaciones de crisis se requiere, tanto para la mujer, hijos e incluso el agresor.

Sin embargo, en España, ni los comedores son gratuitos, ni las madres reciben una prestación económica o paquete con las cosas necesarias para el nacimiento de cada hijo (a escoger por la madre). Ni una prestación económica mensual por cada hijo con independencia del nivel económico, prestación que va aumentando también con el número de hijos y hasta los 17 años de edad; tan solo existen pequeñas prestaciones sujetas a un bajo nivel ingresos o medidas de discriminación positiva en el caso de las familias numerosas. Tampoco las madres tienen opciones suficientes a la hora de volver al trabajo después de haber tenido un hijo/a, las guarderías públicas son muy pocas y las privadas son caras. No existen las opciones de dejar a los hijos con familias cuidadoras o recibir una prestación económica por cuidar a los propios hijos. El material escolar no es gratuito y la educación universitaria tampoco y los jóvenes no reciben ningún tipo de ayuda para independizarse al cumplir 20 años de edad.

Éste es un claro ejemplo de la diferencia de Estado de Bienestar y es que siguiendo las estadísticas publicadas por el Instituto Nacional de 
Estadística, en el año 2001 el coste destinado a políticas de vivienda del total del presupuesto destinado a servicios sociales, fue de un $1 \%$ y el coste del gasto destinado a políticas de familia e infancia fue de un 3\% (http//:www.ine.es).

Sin embargo, y a pesar de todas las diferencias en cuanto a Estado de Bienestar y estatus de la mujer se refiere en ambos países, Finlandia presenta proporcionalmente más casos de violencia doméstica que España. Aunque es importante destacar en este punto que la comparación se ha realizado con diferentes muestras obtenidas de estudios realizados anteriormente en cada país. De cualquier modo, es significativo el alto índice de casos presentados en ambos países, lo que nos lleva una vez a afirmar que la violencia doméstica aparece en todas las sociedades con independencia de raza, etnia, nivel social, cultural o económico (Generalitat Valenciana, Conselleria de Bienestar Social, 2001).

En cuanto a los problemas relacionados con los maltratadores, en ambos casos aparece el alcohol en el primer lugar de la estadística. En Finlandia un $51 \%$ de los agresores tiene problemas de alcohol, mientras que en España afecta al 37\%. En segundo lugar aparecen, desde el punto de vista de los trabajadores sociales, los problemas mentales y, siguiendo las estadísticas realizadas desde las distintas denuncias recibidas en Finlandia, los problemas económicos. En España, en segundo lugar aparecen el estrés y problemas extra-domésticos con un $32 \%$ y en tercer lugar el desempleo con un 13\%. Aunque no debemos olvidar que estos problemas asociados con los maltratadores no son la causa directa de la violencia sino que influyen en mayor o menor medida en el desarrollo de la misma.

\section{TRATAMIENTO OFRECIDO EN FINLANDIA Y EN ESPA- ÑA EN LAS SITUACIONES DE VIOLENCIA DOMÉSTICA.}

En el tratamiento ofrecido en una situación de violencia dómestica también aparecen diferencias entre Finlandia y España.

4.1 En Finlandia, además de todas las prestaciones sociales que reciben los ciudadanos, existen centros de crisis específicos o albergues llamados Hogares para madres e hijos. En los años cuarenta los prejuicios y la resistencia fueron finalmente resueltos, y los primeros hogares para madres solteras y sus hijos comenzaron a establecerse. Los primeros albergues fueron establecidos a finales de los setenta conectados con la existencia de los citados Hogares (Pohjosvirta 2002, 90).

La Federación de Hogares para madres y sus hijos y Alber- 
gues es la organización central donde cada miembro asociado asiste a las familias a través de servicios y proyectos institucionales y comunitarios. El principal objetivo de la Federación es salvaguardar el derecho de los niños para favorecer su crecimiento y un desarrollo seguro, para proveer apoyo a los padres y a la familia entera, y para prevenir la violencia en la familia.

A los Hogares para madres e hijos pueden acceder mujeres embarazadas y el padre puede también entrar en caso de que la situación lo requiera. El tipo de asistencia es acordado con la familia intentando fomentar las relaciones y el cuidado en las relaciones padres-hijo, enseñándoles paso a paso cómo hacer frente a la vida y supervisando la situación después del nacimiento del niño.

Por otra parte los Albergues son centros en los cuales las personas que estén sufriendo una situación de amenazas con violencia familiar pueden encontrar ayuda para resolver la crisis. Todas las partes implicadas en la situación de violencia familiar reciben atención, aunque el objetivo primordial es salvaguardar los intereses de los menores ante todo.

Los Servicios de atención de la comunidad incluyen: trabajador familiar en el hogar de los clientes para enseñar, guiar y controlar a las familias con problemas en el funcionamiento cotidiano de la misma, llamado "Alvari family welfare". También ofrecen un servicio de guardería o cuidados de niños/as pequeños para las familias con mucha presión; asesoramiento y terapia de grupo para hombres violentos o maltratadores; terapia de grupo para las familias, servicio telefónico las 24 horas para situaciones de emergencia y para consejo, discusión y acciones de grupo; lugares o estancias para encuentros entre los miembros de familias separadas y servicios de acogida (vivienda y manutención). Para ello se realiza un acuerdo con la oficina de bienestar social local respecto a la madre e hijos que se acogen. Los servicios de la comunidad están financiados mayormente por los beneficios obtenidos en el negocio de las máquinas tragaperras.

La colaboración con todos los actores locales es un importante factor a mencionar, pues en los talleres de trabajo de red realizados en los albergues están presentes profesionales del servicio de salud, policía y oficinas de bienestar social, además de los trabajadores sociales y demás profesionales del mismo albergue. El trabajo en red es considerado de vital importancia para conseguir de este modo:

- Conocimiento de todos los actores relevantes.

- Mayor información sobre la vida del cliente y de toda la situación de modo global, lo cual hará posible encontrar concretas y más efectivas 
medidas para ayudar y apoyar y llegar a un acuerdo para la solución, donde la institución será responsable en su seguimiento.

- Posibilidades de trabajo como parte de otras instituciones, especialmente cuando éstas realizan visitas en el hogar del cliente.

- Rápida y eficiencia comunicación entre los actores.

- Evitar dobles gastos.

(Tiuraniemi \& Taskinen 2002,96-97).

En estos talleres se discute sobre la intervención en crisis y la terapia para hombres que hayan utilizado la violencia en sus relaciones íntimas afectivas. En Finlandia los albergues para víctimas supervivientes de violencia doméstica trabajan también con los maltratadores. La cooperación y la coordinación entre el trabajador social y las víctimas, niños/as y hombre maltratador es una de las cuestiones principales en este tipo de talleres. Diferentes aspectos de trabajo con los hombres son también cubiertos: ¿Cómo asegurar la seguridad de la víctima durante el contacto y la terapia con su maltratador? ¿Cómo ayudar al hombre maltratador a mantener contacto con sus emociones y cómo ayudarle de manera constructiva para expresar sus emociones? (Säävälä 2002, 96).

4.2. Por otra parte, en España las políticas de igualdad han tenido en consideración la violencia en los últimos años, dedicando un lugar especial a la intervención social. En el III Plan de Igualdad de Oportunidades (1997-2000) (Ministerio Trabajo y Asuntos Sociales), fue introducida una área específica, que más tarde tuvo un extenso desarrollo con la aprobación en 1998 del I Plan de Acción contra la Violencia Doméstica promovido por el Instituto de la Mujer (Ministerio de Trabajo y Asuntos Sociales), en colaboración con los Ministerios de Justicia, Educación, Cultura y Deporte, Salud y Consumo e Interior, y las diferentes Comunidades Autónomas, la Federación de Municipios y Provincias y con las Organizaciones no Gubernamentales.

El I Plan de Acción contra la Violencia Doméstica nació con el objetivo de reducir, en primera instancia, y de erradicar los actos violentos en las familias y arbitrar los recursos necesarios para responder a los efectos en las víctimas. Sin embargo, las estadísticas demuestran que la violencia continúa muy enraizada en la sociedad española y que el número de muertes no disminuye aunque las denuncias aumentan. Por lo tanto, es absolutamente necesario continuar con las acciones contra la violencia y dedicar más recursos para luchar contra la violencia doméstica. Por esta razón, cuando el periodo de validación del I plan finalizó, fue necesario comenzar con un nuevo Plan contra la 
Violencia Doméstica (2001-2004), el cual establece estrategias de desarrollo, con la finalidad de desarrollar los siguientes objetivos:

1. Fomentar la educación basada en los valores del diálogo, respeto y tolerancia para impedir que futuras generaciones puedan reproducir las patrones de comportamiento violento.

2. Mejorar la legislación y el proceso legal, con una mejor protección de la víctima y una penalización más fuerte para el agresor.

3. Completar el mapa de recursos sociales en todo el territorio nacional y potenciar la coordinación entre las acciones de los diferentes organismos e organizaciones sociales, las cuales trabajan para la prevención y eliminación de la violencia doméstica y la asistencia de la víctimas.

Para alcanzar estos objetivos las acciones deben ir articuladas en cuatro grandes áreas: Medidas preventivas y de sensibilización; Medidas legislativas y procedimentales; Medidas asistenciales y de intervención social; Investigación.

Por lo tanto, tal y como se puede observar, España queda muy lejos de plantearse un trabajo con el agresor pues ni si quiera en el II Plan contra la violencia doméstica se menciona la posibilidad de tratamiento con el hombre, más bien ocurre todo lo contrario pues, simplemente, se intenta endurecer las penas como castigo y no como rehabilitación. Es sabido que el tratamiento con los agresores es difícil y no siempre se consigue pero ignorarles es, en cierto modo, permitir que vuelva a ocurrir de nuevo.

Sobre la atención a las víctimas y sus hijos hay que destacar que la descentralización de las Comunidades Autónomas significa también una desigualdad entre unas regiones y otras de España, pues dependiendo del gobierno de cada comunidad se dedicará más o menos presupuesto a la hora de elaborar medidas asistenciales o preventivas para las situaciones de violencia doméstica. Pero, centrándome en la Comunidad Valenciana, los recursos disponibles son los siguientes:

Equipos de Servicios sociales: Éstos están localizados en los ayuntamientos de cada localidad y son el primer paso para la detención, atención y prevención de la violencia contra las mujeres. Los equipos están formados por trabajadores sociales, psicólogos y abogados, trabajando en todo momento en acuerdo con la víctima (Generalitat Valenciana, 2001, 57).

Servicios institucionalizados y especializados para mujeres:

Teléfonos de Urgencia: Si la mujer se encuentra en una situación de violencia de emergencia puede acceder a los teléfonos gratuitos a tra- 
vés de los cuales recibirá información y orientación sobre cómo actuar en este tipo de situaciones. Los teléfonos están disponibles durante las 24 horas del día y cada día del año. El personal que atiende las llamadas son profesionales especializados y están en contacto con diferentes instituciones públicas y privadas, las cuales tienen alguna conexión con este tipo de problemas (juzgados, comisarías, servicios sociales, hospitales, policía, albergues, asociación de mujeres, etc.). Cada mujer que esté sufriendo una situación de violencia será informada sobre los derechos y posibles recursos que puede conseguir para poder tomar la decisión de romper con la situación en la que está envuelta.

InfoDona: Es una red de centros con el objetivo de ofertar información, formación y asesoramiento para mujeres de la Comunidad Valenciana, facilitando su participación, en igualdad de condiciones, en cada ámbito de la región.

Centros Residenciales: El acceso a estos centros es a través de los servicios sociales generales de los ayuntamientos y los preceptores de este servicio son mujeres en situaciones problemáticas o de necesidad y riesgo (desempleadas, mujeres jóvenes con responsabilidades familiares...).

Centros de protección de mujeres o albergues: Pueden acceder solamente mujeres, españolas o extranjeras, con sus hijos/as temporalmente cuando se encuentren en una situación de peligro. Los centros garantizan la máxima seguridad y únicamente los servicios sociales o los centros 24 horas pueden ofrecer la información a cerca de la localización de los mismos. Para acceder a estos centros existe una prioridad en los casos cuando la integridad física o psíquica de la mujer está en peligro, cuando los casos han sido denunciados anteriormente a la policía, cuando la mujer no tiene suficientes recuros económicos y cuando la mujer no tiene otro tipo de apoyo familiar o social.

EMUME: Éste es un servicio de la Dirección General de la Guardia Civil, dirigido a los delitos contra la libertad sexual y violencia física o psicológica sufrida por las mujeres. Este servicio está localizado en la comandancia de la Guardia Civil de cada provincia donde la mujer puede denunciar la situación de violencia.

SAM: Es un servicio de atención a la mujer como una respuesta política articulada por la Policía Nacional para afrontar la violencia sufrida por mujeres. Se encuentra en la Jefatura Superior de Policía de cada provincia donde la mujer puede denunciar su situación.

Centro mujer 24 horas: Es un servicio de la Dirección General de la Mujer adscrito a la Consejería de Bienestar Social de la Generalitat Valenciana. Es un recurso especializado en la atención de mujeres víc- 
timas de violencia física y psicológica, agresiones sexuales, abusos sexuales y acoso sexual en el ámbito laboral. Los centros trabajan durante las 24 horas del día y cada día del año con una atención permanente de trabajadores sociales y psicólogos. Los centros están localizados en las capitales de provincia: Castellón, Valencia y Alicante. En el centro también se elaboran informes anuales y estudios específicos además de crear panfletos y publicaciones sobre temas que pueden contribuir a la formación, información y conciencia de la opinión pública. Por otro lado, también organizan jornadas de formación y conciencia de la opinión pública de los diferentes ámbitos de actuación. En este centro se ofrece además:

1. Servicio gratuito de atención telefónica: para mujeres que demanden ayuda, información u orientación. Y para aquellas que simplemente deseen desahogar sus sentimientos y necesiten ser escuchadas.

2. Servicio de atención directa: la atención puede ser individual o grupal con el objetivo de escuchar y aumentar la autoestima del cliente, además de analizar la situación e identificar las demandas más inmediatas (Generalitat Valenciana, 2001, 57-60).

\section{DISCUSIÓN Y CONSIDERACIONES FINALES.}

En la elaboración del estudio me he centrado en la comparación sobre las estadísticas de Finlandia y España y la influencia que el diferente Estado de bienestar pudiera tener. Claramente, sin embargo, la influencia de diferentes ideologías políticas en diferentes lugares y en tiempos diferentes refleja esta variedad de poder de las fuerzas políticas. La herencia cultural de los diferentes países son un producto de perspectivas ideológicas y debates políticos. La conclusión de los análisis, positivos o negativos, serían útiles para intercambiar información entre ambos países con la intención de mejorar los resultados y ofrecer nuevas alternativas. Tal y como dice Mabbet y Bolderson (1999, 5052):

Estudios detallados de politica pueden producir "insights" teóricos y generalizados. (y...) Quizás el mejor reto en el trabajo comparativo es comprender no sólo la idiosincrasia de las condiciones nacionales sino también los marcos de trabajo conceptuales de los actores en cada pais.

En el estudio de la violencia doméstica en Finlandia y en España he utilizado diferente documentación proporcionada en España por el Instituto de la Mujer además de bibliografía actual sobre el tema. En cuan- 
to al estudio de Finlandia, la universidad de Laponia me ha proporcionado el material suficiente para la realización del mismo además de toda la información facilitada en el centro de prácticas, el Centro móvil de Rovaniemi, donde también la propia experiencia personal vivida durante ese periodo de tiempo con los clientes y profesionales me ha servido de gran ayuda.

El problema presentado a la hora de la investigación y, que resulta necesario mencionar en este punto, ha sido las diferencias existentes en las muestras de las estadísticas de cada país. Por ejemplo, en España la muestra fue recopilada de todas las regiones del país y durante un año, mientras que en Finlandia la muestra fue recopilada en seis ciudades del país y durante un mes. Por lo tanto es difícil generalizar y decir que en Finlandia va a ocurrir lo mismo en cada mes del año. Sin embargo, y teniendo en cuenta que la muestra tampoco se había realizado en todo el país, sino en tan sólo seis ciudades, es probable que de cualquier modo la estadística aumentara en términos de igualdad si se realizara en todas las regiones de Finlandia.

\section{BIBLIOGRAFÍA}

CASILDA BEJAR, R. y TORTOSA, J.M. (1996): Pros y contras del Estado de Bienestar, Madrid, Tecnos.

Conselleria de Bienestar Social (2001): Manual sobre la violencia contra las mujeres. Valencia, Generalitat Valenciana, Consellería de Benestar Social.

DOBASH, T. \& DOBASH, R. (1992): Women, Violence and Social Change. Londres Routledge.

ESPING-ANDERSEN, G. (1990):Three worlds of Welfare Capitalism. Cambridge, Policy Press.

HORLEY, S. (1998): Love and Pain. Londres, BBC Publications.

Instituto de la Mujer: III Plan de Igualdad de Oportunidades (1997-2000). Madrid, Ministerio de Trabajo y Asuntos Sociales.

Instituto de la Mujer: I Plan de Acción contra la Violencia Doméstica (19982000) Madrid, Ministerio de Trabajo y Asuntos Sociales.

Instituto de la Mujer: II Plan de Acción contra la Violencia Doméstica (20012004). Madrid, Ministerio de Trabajo y Asuntos Sociales.

Instituto de la Mujer (2001): Manual sobre la violencia contra la mujer. Madrid, Ministerio de Trabajo y Asuntos Sociales.

JONES, C. (1993): New perspectives on the welfare state in Europe. Londrres, Rouletdge. 
MABBET, D. \& BOLDERSON, H. (1999): Journal of Public Policy, vol.18, p $177-200$

MORENO, L. (2001): "La "vía media" española del modelo de bienestar mediterráneo", Papers 63/64.

PHJOSVIRTA, R. (2002): "Roots and development of the Finnish crisis centre institution", en Crisis centres and violence against women. Arkhangelsk, Ministry of Education of Russian Federation- Pomor State UniversityM.V.Lomonosov Centre of gender researches and Oulu University, págs. 90-91.

PICÓ, J.(1996): "Modelos sobre el Estado del Bienestar", en Casilda Bejar, R. y Tortosa, J.M. Pros y contras del Estado de Bienestar, Madrid, Tecnos.

PRYKE, J. \& THOMAS, M. (1998): Domestic Violence and Social Work. Ashgate, England.

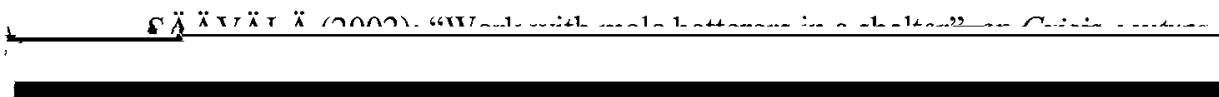

and violence against women. Arkhangelsk, Ministry of Education of Russian Federation- Pomor State University- M.V.Lomonosov Centre of gender researches and Oulu University, pág. 96.

SUMMERS, R. W. \& HOFFMANA. M. (2002): A world view of social issues. Westport Greenwood Press.

TIURANIEMI, H. y TASKINEN, S. (2002): "Collaboration with local actors", en Crisis centres and violence against women. Arkhangelsk, Ministry of Education of Russian Federation- Pomor State University- M.V.Lomonosov Centre of gender researches and Oulu University, págs. 96-97.

http://www.virutal.finland.fi/finfo/English/women.html

http://www.ine.es 